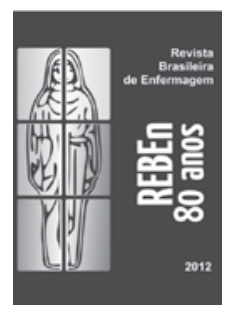

\title{
Pós-Graduação Stricto Sensu em Enfermagem no Brasil: avanços e perspectivas
}

\author{
The Strict Sense Nursing Postgraduation in Brazil: advances and perspectives \\ El Postgrado Stricto Sensu en Enfermería en Brasil: avances y perspectivas
}

\section{Carmen Gracinda Silvan Scochi', Denize Bouttelet Munari", Francine Lima Gelbcke"II, Alacoque Lorenzini Erdmann', Maria Gaby Rivero de Gutiérrez ${ }^{\mathrm{IV}}$, Rosalina Aparecida Partezani Rodrigues ${ }^{v}$}

\author{
' Universidade de São Paulo, Escola de Enfermagem de Ribeirão Preto, \\ Departamento de Enfermagem Materno-Infantil e Saúde Pública. Ribeirão Preto-SP, Brasil. \\ "Universidade Federal de Goiás, Faculdade de Enfermagem. Goiânia-GO, Brasil. \\ II' Universidade Federal de Santa Catarina, Centro de Ciências da Saúde. Florianópolis-SC, Brasil. \\ Iv Universidade Federal de São Paulo, Departamento de Enfermagem. São Paulo-SP, Brasil. \\ ${ }^{v}$ Universidade de São Paulo, Escola de Enfermagem de Ribeirão Preto, \\ Departamento de Enfermagem Geral e Especializada. Ribeirão Preto-SP, Brasil
}

Submissão: 06-08-2013 Aprovação: 07-08-2013

\begin{abstract}
RESUMO
A Enfermagem é campo de conhecimento específico e prática social que se consolida e se fortalece como ciência. No Brasil, tem se desenvolvido em função do aumento e qualificação dos programas de pós-graduação stricto sensu. Este artigo tem como objetivo apresentar um resgate histórico da Pós-Graduação Stricto Sensu de Enfermagem no Brasil e refletir sobre sua evolução, avanços, desafios e perspectivas futuras. Explora-se a criação dos cursos de pós-graduação stricto sensu, destacando os movimentos para se construir a cultura da pós-graduação acadêmica e profissional na Enfermagem. Apresenta-se a trajetória histórica da sua consolidação, expansão, conquista da excelência e visibilidade internacional ao longo de quatro décadas e apontam-se os desafios e as perspectivas futuras. Constata-se que a pós-graduação na área vem contribuindo para o avanço e consolidação do conhecimento científico, tecnológico e de inovação em Enfermagem e saúde, tendo como filosofia, o respeito à diversidade e o livre debate de ideias, a elevação da qualidade de vida e saúde e a efetivação do exercício da cidadania.

Descritores: Educação de Pós-Graduação em Enfermagem; Pesquisa em Enfermagem; Enfermagem.
\end{abstract}

\begin{abstract}
Nursing is a specific field of knowledge and social practice that has been consolidated and strengthened as science. In Brazil, it has been developed due to the increase and qualification of strict sense post-graduate programs. This study aims to present a historical review of the strict sense post-graduate nursing courses in Brazil and to reflect on their evolution, progress, challenges and future perspectives. It explores the creation of strict sense post-graduate courses, highlighting the movement to build a culture of academic and professional post-graduation in nursing. The historical path of their consolidation, expansion, conquest of excellence and international visibility over four decades, and the challenges and future perspectives are showed. It is found that the post-graduate programs in the field has contributed to the advancement and consolidation of scientific, technological knowledge and innovation in nursing and health care, having as philosophy the respect for diversity and the free exchange of ideas, the improvement of quality of life and health, and the effectiveness of citizenship.
\end{abstract}

Key words: Nursing Post-Graduate Education; Nursing Research; Nursing. 


\section{RESUMEN}

La Enfermería es un campo específico de conocimiento y práctica social que se consolida y fortalece como ciencia. En Brasil, se ha desarrollado debido al aumento y cualificación de los programas de postgrado stricto sensu. Este estudio tiene como objetivo presentar una revisión histórica del Postgrado Stricto Sensu en Enfermería en Brasil y reflexionar sobre su evolución, progresos, desafíos y perspectivas futuras. Se explora la creación de cursos de postgrado stricto sensu, destacando el movimiento para construirse una cultura de posgrado académico y profesional en Enfermería. Se presenta la trayectoria histórica de su consolidación, expansión, conquista de la excelencia y visibilidad internacional a lo largo de cuatro décadas, y se señalan los desafíos y perspectivas futuras. Se constató que el postgrado en la área ha contribuido para el avance y consolidación del conocimiento científico, tecnológico y de innovación en Enfermería y salud, y teniendo como filosofía el respecto a la diversidad y el libre debate de ideas, el aumento de la calidad de vida y salud y la efectuación del ejercicio de la ciudadanía. Palabras clave: Educación de Postgrado en Enfermería; Investigación en Enfermería; Enfermería.

\section{AUTOR CORRESPNDENTEＣarmen Gracinda Silvan ScochiＥ-mail: carmenscochi@gmail.com}

\section{INTRODUÇÃO}

A Enfermagem como um campo de domínio específico de conhecimentos e saberes e como profissão social, vem marcando história na sua evolução conquistando espaços e consolidando áreas temáticas e linhas de pesquisa mediante a formação de recursos humanos altamente qualificados e com atuação e domínios em várias dimensões e níveis de complexidade do cuidado a saúde.

A Associação Brasileira de Enfermagem, em comemoração aos 80 anos da Revista Brasileira de Enfermagem, brinda sua comunidade acadêmica e da prática em campo com um número especial que registra a história e trajetória dos atores sociais que contribuíram para a qualificação deste importante periódico. Assim, buscando informações junto a suas lideranças que marcaram representação da área junto à Coordenação de Aperfeiçoamento de Pessoal de Nível Superior - CAPES, objetiva-se com este artigo apresentar um resgate histórico da Pós-Graduação Stricto Sensu de Enfermagem no Brasil e refletir sobre sua evolução, avanços, desafios e perspectivas futuras.

A Pós-Graduação Stricto Sensu em Enfermagem surge na América Latina em 1969 com a criação dos primeiros mestrados acadêmicos na Venezuela e Colômbia e em 1972, no Brasil, em resposta à necessidade de qualificar enfermeiros para sua inserção no mercado de trabalho, em instituições de ensino, de pesquisa ou de prestação direta de serviços. Os primeiros doutorados em Enfermagem foram criados, no Brasil, em 1982, na Venezuela em 1999, no México em 2003 e na Colômbia em 2004, seguindo os demais países na conquista do seu primeiro doutorado em Enfermagem(1). Já nos Estados Unidos da América, os primeiros mestrados e doutorados surgiram a partir da década de $1930^{(2)}$.

Os avanços da pesquisa científica vêm sendo concretizados quase que totalmente nos espaços ou ambientes onde se efetivam os cursos de mestrado acadêmico, mestrado profissional e doutorado. Estes são produzidos em meio às atividades acadêmicas, centradas nos grupos de pesquisa, que integram alunos de todos os níveis de formação - da graduação ao estágio pós-doutoral - com vínculos e procedências diversas.

Na pós-graduação da Enfermagem brasileira essa também é uma realidade que é orientada por políticas que propiciam novas conquistas, fortalecem e trazem inovações em suas ações para o alcance de impactos educacionais, sócio-políticos e científicos/tecnológicos significativos para a Enfermagem e Saúde, como ciência, tecnologia e profissão social.

Reconhecemos que o trabalho da Coordenação de Área é um compromisso coletivo, balizado pela filosofia e políticas da CAPES e dos Programas de Pós-Graduação em Enfermagem. Seu desafio é viabilizar ações para crescimento e fortalecimento da pós-graduação brasileira e da pesquisa científica, incrementando a formação qualificada de mestres e doutores e a produção de conhecimentos avançados. Ilustrar a trajetória desse trabalho é o que se pretende no presente artigo.

\section{PÓS-GRADUAÇÃO EM ENFERMAGEM NO BRASIL: SUR- GIMENTO E CONSOLIDAÇÃO}

A pós-graduação brasileira, instituída pela Lei de Diretrizes e Bases da Educação Nacional em 1961 e aprovada pelo ConseIho Federal de Educação em 1965, surgiu em um contexto político de promoção do desenvolvimento econômico do país, o que demandava a formação de recursos humanos qualificados com vistas a atender duas demandas principais, quais sejam: "a necessidade futura de mão-de-obra especializada para preencher os novos empregos criados pelo desenvolvimento previsto e a necessidade de cientistas, pesquisadores e técnicos, aptos a desenvolver a pesquisa, indispensável à mudança"(3).

Cabe destacar que, desde a instituição dos cursos de pós-graduação, houve a preocupação com a avaliação do seu desempenho, cabendo à Coordenação de Aperfeiçoamento de Pessoal de Nível Superior (CAPES) a responsabilidade do acompanhamento e avaliação dos mesmos.

Nessa mesma época, precisamente no início dos anos 1970, duas docentes da Escola de Enfermagem Anna Nery, da Universidade Federal do Rio de Janeiro - UFRJ, foram designadas como assessoras da CAPES, com vistas à mobilização das Escolas de Enfermagem para discutir os interesses da área em relação aos cursos de pós-graduação(4). Nesse cenário, em 1972, nessa Escola, iniciou-se o primeiro curso de mestrado no país. Vale destacar que a primeira turma de alunas era composta, principalmente, por professoras de Escolas de Enfermagem do Rio de Janeiro e São Paulo, cuja finalidade era 
formar mestres de diferentes regiões para que estas pudessem expandir a pós-graduação da área.

Após a implantação do primeiro curso de mestrado, e em consonância com a política desenvolvimentista da época, mais oito cursos de mestrado foram criados na década de 1970. Foram eles: Escola de Enfermagem da USP (1973 - Enfermagem), Escola de Enfermagem de Ribeirão Preto - EERP/ USP (1975 - Enfermagem Psiquiátrica e 1979 - Enfermagem Fundamental), Universidade Federal de Santa Catarina e Universidade Federal do Rio Grande do Sul (1976), Departamento de Enfermagem da Escola Paulista de Medicina (1978), Universidade Federal da Bahia e Universidade Federal da Paraíba, ambos em 1979. Verifica-se que a maioria dos cursos estava concentrada na região sudeste, porém as regiões sul e nordeste também tiveram um papel importante nesse caminhar histórico da Pós-Graduação em Enfermagem.

Na década seguinte (1980 a 1989) houve uma menor expansão dos cursos de mestrado, sendo criado apenas um, na Universidade do Rio de Janeiro, em 1982. Por outro lado, essa década representou um marco para a Pós-Graduação em Enfermagem, pois foram criados quatro programas de doutorado, sendo o primeiro, o Doutorado USP Interunidades, criado em 1981 e iniciado em 1982, fruto da conjunção de esforços de docentes das Escolas de Enfermagem da USP e de Ribeirão Preto. Os outros programas foram os da Universidade Federal do Estado de São Paulo - UNIFESP (1986), da EE/USP e da UFRJ (1989).

Nessa mesma década, em meio a mudanças no paradigma da atenção à saúde no país, a Enfermagem também propõe mudanças nas suas ações que, mesmo ainda incipientes, conseguiram conquistar reconhecimento para desenvolver um projeto educacional e de pesquisa na pós-graduação stricto sensu, graças aos esforços das líderes da área na época. Tais conquistas se basearam em ações que buscavam responder às demandas exigidas pelo avanço da ciência e tecnologia na área da saúde, dos novos paradigmas de atenção à saúde e dos novos padrões de avaliação de qualidade dos programas de pós-graduação.

Foi neste cenário de mudanças que a Enfermagem foi instituída como área de conhecimento específica, com representação no Conselho Técnico Científico (CTC) da CAPES, por meio da Resolução no 1, de 7 de abril de 1987. Embora não tivesse tanta autonomia, porque era uma representação dentro de uma área que agregava Odontologia, Saúde Coletiva, Educação Física, entre outras, teve sua primeira representante da área ${ }^{(4)}$.

Na década de 1990, o ensino superior enfrentou sérias dificuldades decorrentes da redução progressiva do orçamento, bem como do esvaziamento dos seus quadros docentes, em consequência da reforma da previdência social que estimulou muitas aposentadorias precoces. Essa conjuntura repercutiu duramente na Enfermagem, pois dificultou o deslocamento de docentes para cursarem a pós-graduação, situação agravada pela falta de uma política institucional explícita de formação de recursos humanos. Diante dessa situação, as Escolas de Enfermagem e a representação da área na CAPES mobilizaram-se no sentido de fazer face às dificuldades enfrentadas, buscando formas alternativas para viabilizar a formação pós-graduada de recursos humanos qualificados.

Um dos resultados dessa mobilização foi a criação, em
1992, da Rede de Pós-Graduação em Enfermagem da Região Sul (REPENSUL), sob a coordenação da UFSC, que atendeu a formação de docentes de seis universidades federais e a extensão do curso de mestrado para três localidades da região. Além disso, foram criados 11 cursos de pós-graduação, dos quais, seis eram de mestrado - EERP/USP - Enfermagem em Saúde Pública (1991), UFC (1993), UFMG (1994), UERJ e UNICAMP (1999), e, a reativação do mestrado da UFRGS (1998). Além desses, cinco novos cursos de doutorado resultaram da expansão dos mestrados na EERP/USP - Enfermagem Fundamental (1991), UFSC (1993), EERP/USP - Enfermagem em Saúde Pública e UFC (1998) e EERP/USP - Enfermagem Psiquiátrica (1999).

Outro fato importante a ser destacado nesta década foi a mudança na sistemática de avaliação da CAPES, caracterizada pelo aumento do rigor nos critérios de avaliação, alteração da periodicidade das avaliações, passando de bienal para trienal, avaliação por programa e não mais por cursos isoladamente e substituição dos conceitos atribuídos por uma escala numérica de 1 a 7, além da adoção de critérios para classificação da qualidade dos periódicos científicos de todas as áreas de conhecimento, criando o sistema denominado Qualis/CAPES.

Todas essas mudanças tiveram um forte impacto nos programas de pós-graduação em Enfermagem, gerando um grande esforço por parte de todos os envolvidos para que não houvesse prejuízos. Por outro lado, a pressão exercida sobre a área teve seu lado positivo, pois houve um crescimento significativo do número de programas e de titulados, bem como redução do tempo de titulação. Nessa época, a Enfermagem contava com 25 cursos de pós-graduação, sendo dezesseis (16) de mestrado e nove (9) de doutorado.

No final do século $X X$, a área iniciou uma nova era de avanço da pós-graduação, com expansão do número de cursos novos, principalmente, em diferentes regiões do país, fruto dos novos doutores formados nos programas das regiões sul e sudeste e o início do mestrado profissional. As universidades brasileiras também expandiram suas colaborações na formação de doutores da área da Enfermagem em diferentes países, como Argentina, Chile, Colômbia, Peru, Venezuela, México, Costa Rica, Panamá, Bolívia, Angola, Moçambique, dentre outros. Esses novos doutores foram responsáveis pela implementação de programas de pós-graduação em Enfermagem, em seus países de origem.

\section{EXPANSÃO DOS PROGRAMAS DE PÓS-GRADUAÇÃO NA ÁREA DE ENFERMAGEM: EM BUSCA DA EXCELÊNCIA}

Um novo marco iniciava-se no século XXI, a era da comunicação com grandes avanços tecnológicos e de inovação nos sistemas de informação e computação. Esta nova forma de trabalhar se expandiu, influenciando diretamente a formação em pós-graduação, pois permitiu às universidades e aos pesquisadores conectar-se com os "outros", por meio de acessos mais fáceis e rápidos.

A Enfermagem também acompanhou essa mudança que, na primeira década do século XXI, teve ainda forte investimento em políticas e estratégias que promovessem a elevação da qualidade dos Programas. Este se caracterizou pelo 
estabelecimento de critérios balizados por políticas centradas na valorização do docente-pesquisador, na formação de doutores com perfil de pesquisador e produção científica de alto impacto e inserção internacional. Também fizeram parte desse movimento o desenvolvimento das diferentes regiões do país na perspectiva de fortalecimento e soberania do mesmo, com aumento da visibilidade e reconhecimento internacional.

Nesse início de século, a Enfermagem passou a ter espaço como membro do Conselho Técnico Científico (CTC) da CAPES, por meio da instituição da Coordenação de Área, o que possibilitou sua atuação junto a outras áreas, discutindo temas que permitissem maior expansão em quantidade e qualidade da pesquisa na área. Assim, foi possível para a área discutir a relevância da Política Nacional de Pós-Graduação e, sobretudo, a participação conjunta na Grande Área da Saúde.

Dentre as diversas iniciativas dos Representantes e de Coordenadores de Programas de Pós-Graduação destacam-se a elaboração de Planos de Trabalho da Área da Enfermagem, de 2001 a 2010, e os Relatórios da avaliação trienal de 2001$2003^{(5)}$ e de 2004-2007(6) . Para o triênio 2007-2009, foi instituído o Documento de Área $2009^{(7)}$, no qual se fazia referência à trajetória e ao acompanhamento da evolução das gestões anteriores nesta Coordenação. A intensificação da produção científica e social da Enfermagem brasileira no contexto nacional e internacional, bem como a captação de recursos financeiros para bolsas de estudo, auxílios à pesquisa, por meio de diversos editais e de melhorias de infraestrutura física, de pessoal, de material e apoio logístico também permitiram alterar os parâmetros de alta qualificação e publicação adequada.

As especificidades da Área de Enfermagem foram marcadas nesse Documento de Área ${ }^{(7)}$, o qual descreve as Considerações gerais sobre o estado atual da Área com a definição do Perfil do Doutor em Enfermagem, seguindo com Considerações gerais sobre a Ficha de Avaliação para o triênio 2007-2009 e os Novos critérios para classificação dos periódicos com publicações da área. O documento é finalizado com o detalhamento dos critérios de avaliação amplamente trabalhados nesse período.

Outro documento produzido pela área também importante neste período foi o Relatório da avaliação trienal de 2007-2009(8), no qual se destaca a apresentação das Políticas para o trabalho da Coordenação da Área de Enfermagem da Capes para o triênio 2007/2009, o processo de construção de Critérios para Classificação de Livros da Enfermagem, os critérios e indicadores para os Programas de Excelência notas 6/7, dentre outros.

Destaca-se nesse documento o posicionamento da área com relação à formação de seus pesquisadores ou cientistas que é orientada por um Perfil do Doutor em Enfermagem, visando ao alcance da excelência, espelhada no perfil internacional da formação de doutores da área, tendo várias competências/aptidões/domínios ${ }^{(8)}$.

Ao final da primeira década do século XXI, a Área de Enfermagem do Brasil se despontou no mundo, chegando a $12^{\mathrm{a}}$ e $13^{\mathrm{a}}$ no ranking de produção científica, divulgado no http://www.scimagojr.com/countryrank.php. Com isso, os programas conquistaram o pleito à nota 6 , mediante o crescimento de publicações mais qualificadas, ganhando espaço e responsabilidade de sustentação dessa inserção, como reflexos dos esforços no investimento na inserção e visibilidade internacional ${ }^{(8)}$.

Também foram possíveis nesse período avanços na definição de métricas e políticas para a avaliação, nos sistemas de registros do desempenho dos programas, no processo de acompanhamento, na infraestrutura física e pessoal da CAPES, bem como em outros aspectos que contribuíram para a busca da excelência.

A expansão da área em número de cursos é destacada nos dados sobre o percentual de crescimento dos programas de pós-graduação - PPG/cursos aprovados: em dezembro de 2007 contávamos com 32 PPG/42 cursos (27 Mestrados Acadêmicos - MA, 13 Doutorado - DO e 02 Mestrados Profissionais - MP) e em junho de 2011 chegamos a 51 PPG/75 cursos (42MA, 24DO e 09MP), ou seja, um aumento de 59,3\% e 78,5\% em número de programas e cursos, respectivamente. Do mesmo modo, o crescimento dos programas segundo a distribuição dos conceitos: 51 PPG (19MA, 22DO, 01DO, 01MA/DO/MP e 08MP) sendo 23 PPG nota 3, 15 PPG nota 4, 09 PPG nota 5 e 04 PPG nota 6 . E, dentre os 75 cursos: 42 MA (17 nota 3, 13 nota 4, 08 nota 5 e 04 nota 6$) ; 24$ DO (11 nota 4, 09 nota 5 e 04 nota 6); e 09 MP (06 nota 3 e 03 nota 4). Com isso, também foi significativo o crescimento em número de alunos titulados.

Além do número de programas, verifica-se o crescimento da qualidade da formação de recursos humanos e do desenvolvimento de pesquisas. A avaliação dos Programas de Pós-Graduação da Enfermagem segue os critérios da Grande Área da Saúde da CAPES, o que tem permitido sua visibilidade social, tanto nacional, quanto internacional, por meio de parcerias e convênios entre as universidades brasileiras e estrangeiras.

A década de 2000 a 2010 foi marcada por saltos significativos, fruto do esforço centrado em políticas e estratégias da CAPES, dos programas de pós-graduação da área, do esforço das lideranças da Enfermagem, representantes em diversos órgãos e espaços políticos da área, docentes, pesquisadores, alunos, editores de revistas, comunidade científica, órgãos de classe e outros.

\section{CENÁRIO ATUAL: VISIBILIDADE INTERNACIONAL E DESAFIOS}

A Pós-Graduação em Enfermagem no Brasil, no auge de seus 40 anos, encontra-se em franca expansão constatada pelo aumento do número de cursos e programas, de egressos e da produtividade científica com publicação de artigos em periódicos com fator de impacto.

Buscando manter os espaços conquistados, a área permanece atenta às diretrizes da CAPES para o desenvolvimento da pós-graduação, sobretudo, vislumbrando as recomendações e desafios propostos no Plano Nacional de Pós-graduação PNPD 2011-2020(9).

Nessa busca constante de conquista da excelência, cabe destacar que, na avaliação trienal de 2010, nove programas apresentaram desempenho muito bom e liderança nacional (nota 5) e quatro tiveram reconhecida sua excelência e inserção internacional (nota 6).

Mais recentemente, foram aprovados mais 12 cursos, sendo quatro doutorados e oito mestrados (05 acadêmicos e 03 profissionais). Desse modo, em maio de 2013, a área contava 
com 63 programas de pós-graduação stricto sensu (26MA e DO, 02DO, 21MA e 14MP), totalizando 89 cursos (28DO, 47MA e 14MP), assim distribuídos por região: 2,3\% (02MA) no Norte; 20,2\% (05DO, 11MA e 02MP) no Nordeste; $9,0 \%$ (02DO, 05MA e 01MP) no Centro-Oeste; 49,4\% (15DO, $21 \mathrm{MA}$ e 08MP) no Sudeste e 19,1\% (06DO, 08MA e 03MP) no Sul. Esse quantitativo de cursos stricto sensu da Área de Enfermagem representava 1,7\% do total de cursos de pós-graduação brasileiros e 9,7\% dos da área de Ciências da Saúde.

De acordo com o desempenho na avaliação, tais cursos/ programas estão assim distribuídos: $29(45,3 \%)$ nota 3; 22 $(34,4 \%)$ nota $4 ; 09(14,1 \%)$ nota 5 e $04(6,2 \%)$ nota 6 . Justifica-se aqui o total de 64 notas de cursos/programas pelo fato de o mestrado da Universidade Federal de Pelotas ter tido nota 3 na trienal 2007/2009 e, em 2012, ter obtido aprovação para o doutorado, com nota 4 . A concentração de cursos nota 3 justifica-se pelo crescimento de mestrados novos; todavia, tem-se perspectiva de mudança nessa distribuição, pois quatro (4) novos mestrados profissionais foram aprovados com nota 4 e, apesar de ainda não se ter programa nota 7, há tendência de ampliar o reconhecimento da excelência e inserção internacional de alguns programas da Área de Enfermagem.

Esta inserção internacional pode ser observada em função de parcerias consolidadas entre os programas brasileiros e os centros de excelência internacional, como a University of Alberta - CA, McGill University - CA, University of Toronto - CA, University of Pennsylvania - USA, University of Washington USA, University of North Carolina Chapel Hill - USA, King's College London University of London - UK, dentre outros.

Destaca-se, ainda, que a expansão da pós-graduação na área é caracterizada pela cobertura em todo o território nacional, embora nas regiões Norte e Centro-Oeste ainda haja carência de oferta desse nível de ensino, com a qualidade exigida pela Área de Enfermagem e CAPES. Assim, permanecem como desafios a redução da assimetria no desenvolvimento da pós-graduação da Enfermagem brasileira e também a sustentabilidade dos cursos/programas novos assegurando a qualidade da formação pretendida ${ }^{(10)}$.

A Figura 1 mostra o crescimento da pós-graduação na área, em especial na última década.

No que se refere à titulação de mestres e doutores houve aumento gradual, perfazendo mais de 500 defesas anuais de mestrado e doutorado nos programas da Área de Enfermagem, desde 2006, como ilustra a Figura 2. Permanece o desafio de expandir a titulação de doutores em Enfermagem, pois seu crescimento tem sido insuficiente para atender a necessidade do mercado de trabalho e está aquém da meta de duplicar o número de pesquisadores qualificados, em 10 anos, estabelecida no PNPD 2011-2020

O impacto deste crescimento da pós-graduação se evidencia também na melhoria da produção científica, com aumento expressivo no número de documentos indexados na base Scopus/SCImago e, consequentemente, no ranking mundial, em que a Enfermagem brasileira ocupava o $25^{\circ}$ lugar da produção da área em 2005 e ascendeu para o $6^{\circ}$ lugar em 2010, superado pelos Estados Unidos da América, Reino Unido, Austrália, França e Canadá.

No âmbito nacional, a Enfermagem representava 0,23\% do conhecimento científico divulgado nessa base de dados em 2005, passando a 1,87\% em 2010, portanto, com crescimento relativo de $713 \%$ e muito superior àquele ocorrido nas Ciências Sociais e Medicina, conforme mostra a Tabela 1.

Figura 1 - Evolução da Pós-Graduação na Área de Enfermagem no Brasil (1972-2013).

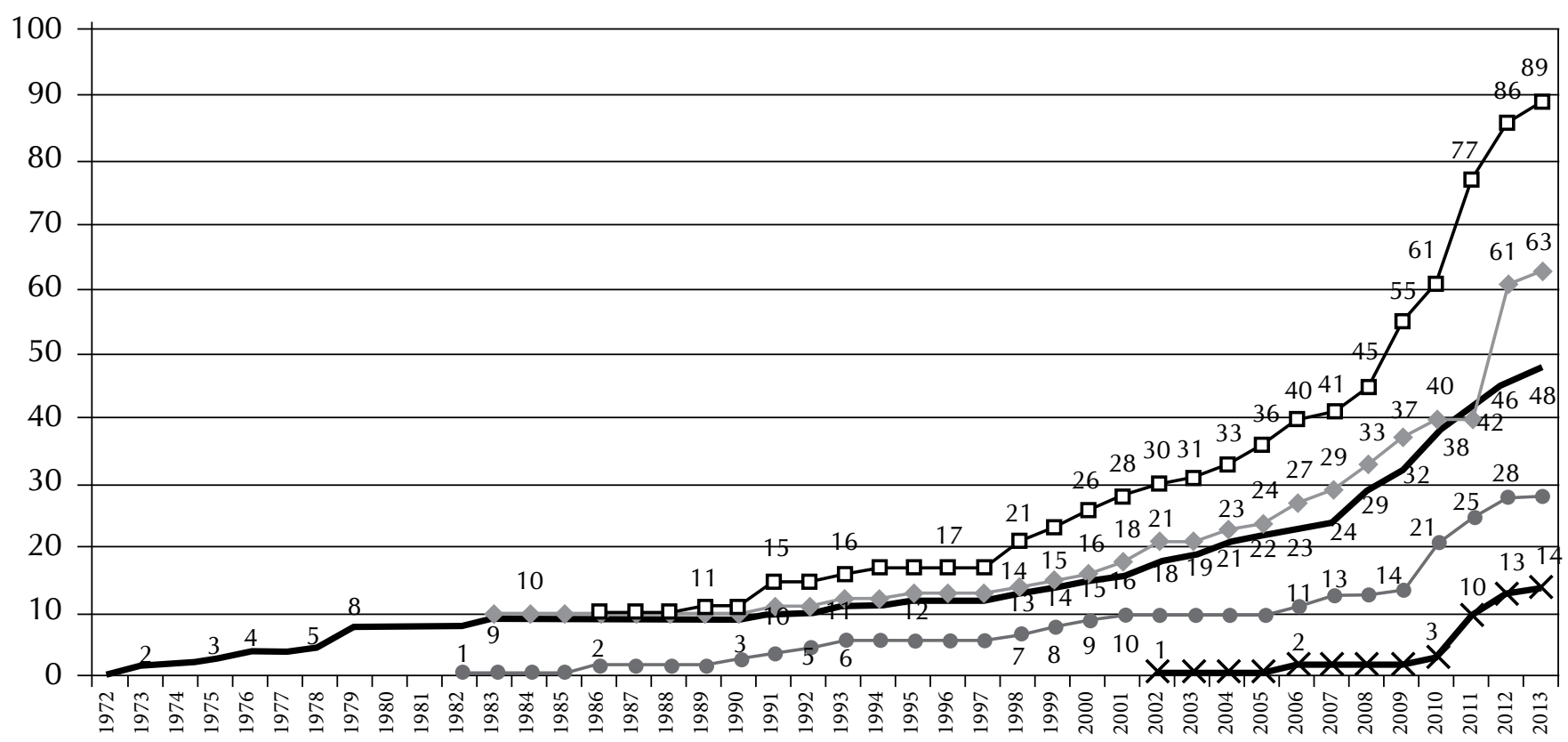

\begin{tabular}{|c|c|c|}
\hline - N No Cursos & $\multimap N^{\circ} P G$ & $\longrightarrow$ MA \\
\hline
\end{tabular}


Figura 2 - Alunos titulados nos Programas de Pós-Graduação da Área de Enfermagem (1998-2011).

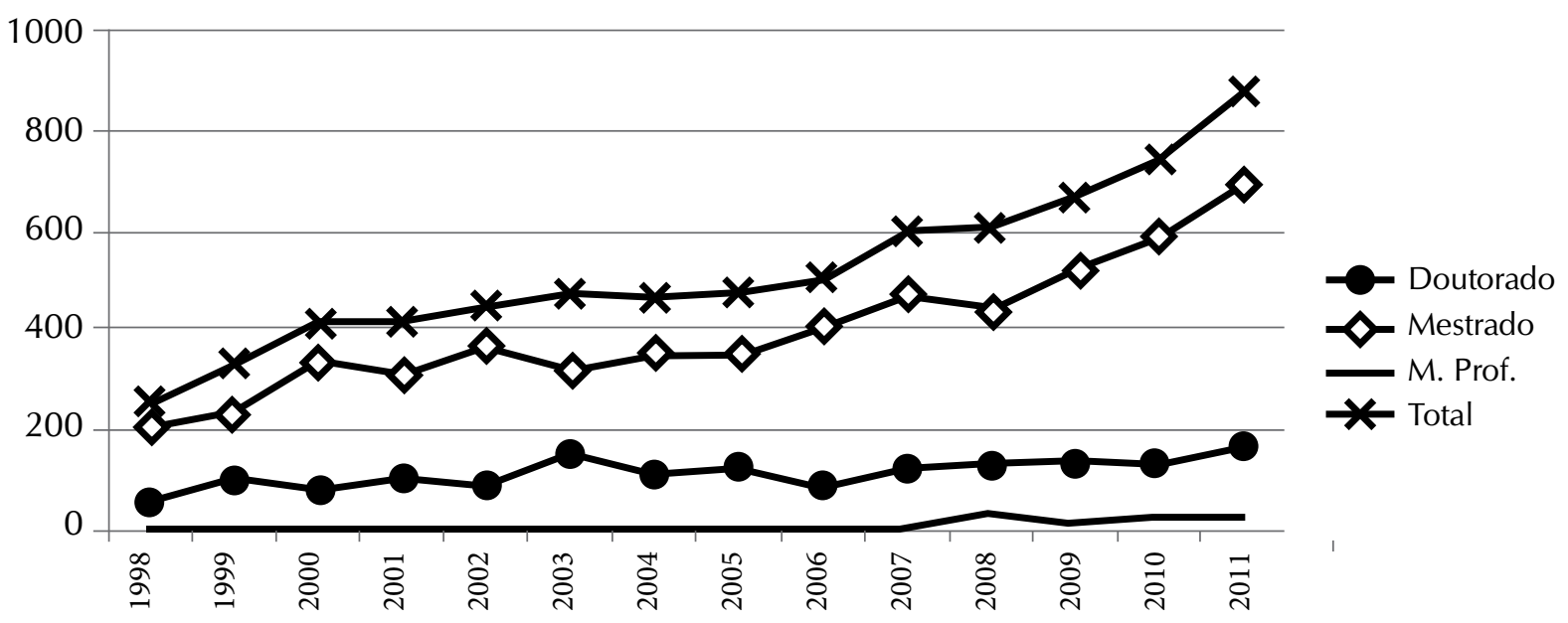

Esse crescimento e visibilidade da produção científica no cenário nacional e internacional é fruto da expansão da pós-graduação em Enfermagem nos últimos anos, uma vez que em 2005 a área tinha 33 cursos aprovados (10DO, 22MA e 01MP), aumentando para 62 (21DO, 38MA e 03MP) cursos em 2010.

Assim, a dimensão da internacionalização da pós-graduação na área é resultado, principalmente, da qualidade da produção científica dos docentes permanentes e discentes ou egressos, tendo como aspectos principais a publicação de resultados das pesquisas em periódicos indexados, com fator de impacto e de referência para a Enfermagem mundial, bem como o reconhecimento internacional pelos pares, evidenciado pelas citações das publicações de docentes, discentes e egressos dos programas de pós-graduação ${ }^{(10)}$.

O reconhecimento do crescimento qualitativo da pesquisa em Enfermagem ainda pode ser considerado pela ampliação do número de revistas indexadas nas principais bases nacionais e internacionais e de referência para as áreas de Enfermagem e da Saúde, além do aumento dos índices censiométricos. Ressalta-se que quatro periódicos de Enfermagem, editados no Brasil, estão na Web of Science, três dos quais obtiveram WoS/ JCR com fatores de impacto equivalentes ao de outras revistas editadas nos Estados Unidos, de referência internacional para a área. Ainda, oito periódicos brasileiros da área estão indexados na base Scopus/SCImago com SJR e índice $\mathrm{H}$.

Outras indexações importantes em bases referenciais são: quatro periódicos brasileiros na MEDLINE - National Library of Medicine, seis no CINAHL - Cumulative Index to Nursing \& Allied Health Literature, dez no CUIDEN que disponibiliza o Índice das Revistas sobre Cuidados de Salud con Repercusión en Iberoamérica, sete no SciELO - Scientific Electronic Library Online, 19 na Literatura Latino-americana e do Caribe

Tabela 1 - Crescimento relativo (CR) da produção científica brasileira em três áreas de conhecimento. SCImago 2005/2007/2009/2010.

\begin{tabular}{|c|c|c|c|c|c|c|c|c|}
\hline \multirow{2}{*}{ Áreas } & \multicolumn{2}{|c|}{2005} & \multicolumn{2}{|r|}{2007} & \multicolumn{2}{|c|}{2009} & \multicolumn{2}{|l|}{2010} \\
\hline & $\mathrm{N}^{\circ}$ & $\%$ & $\mathrm{~N}^{\circ}$ & $\%$ & No & $\%$ & $\mathrm{~N}^{\circ}$ & $\%$ \\
\hline \multirow{3}{*}{$\begin{array}{l}\text { Medicina } \\
\text { CR } 2 \text { anos } \\
\text { CR } 5 \text { anos }\end{array}$} & 5619 & 24,91 & 7866 & 25,24 & 10499 & 25,40 & 11957 & 26,46 \\
\hline & & $1,3 \%$ & & $0,6 \%$ & & $4,2 \%$ & & \\
\hline & \multicolumn{8}{|c|}{$6,2 \%$} \\
\hline \multirow{3}{*}{$\begin{array}{l}\text { Enfermagem } \\
\text { CR } 2 \text { anos } \\
\text { CR } 5 \text { anos }\end{array}$} & 51 & 0,23 & 397 & 1,27 & 646 & 1,56 & 845 & 1,87 \\
\hline & & $452,2 \%$ & & $22,8 \%$ & & $19,9 \%$ & & \\
\hline & \multicolumn{8}{|c|}{$713 \%$} \\
\hline \multirow{3}{*}{$\begin{array}{r}\text { Ciências Sociais } \\
\text { CR } 2 \text { anos } \\
\text { CR } 5 \text { anos }\end{array}$} & 247 & 1,09 & 1052 & 3,38 & 1519 & 3,67 & 1770 & 3,92 \\
\hline & & $210,1 \%$ & & $8,6 \%$ & & $6,8 \%$ & & \\
\hline & \multicolumn{8}{|c|}{$259,6 \%$} \\
\hline Total (todas áreas) & \multicolumn{2}{|c|}{22.558} & \multicolumn{2}{|c|}{31.162} & 41.334 & & \multicolumn{2}{|l|}{45.189} \\
\hline CR 5 anos & \multicolumn{8}{|c|}{$100,3 \%$} \\
\hline
\end{tabular}


em Ciências da Saúde - LILACS e 16 na Rev@Enf da Biblioteca Virtual de Saúde-Enfermagem (BVS/Enf) que inclui uma coleção das melhores revistas de Enfermagem de diferentes países (Brasil, Espanha, Portugal, Cuba, Colômbia e Chile), publicadas na metodologia SciELO. A grande maioria desses periódicos está vinculada a universidades com programas de pós-graduação em Enfermagem.

Esse cenário evidencia o reconhecimento da qualidade da ciência da Enfermagem brasileira, comparável aos padrões de excelência internacional da área e a conquista de espaços políticos com maior participação dos enfermeiros pesquisadores em órgãos de fomento, a exemplo da Coordenação de Área na CAPES e o Comitê Assessor de Enfermagem no CNPq, assim como nos processos decisórios em instituições e associações de editoração nacional e internacional, com impactos na internacionalização da produção da Enfermagem brasileira e na melhoria da qualidade editorial dos periódicos da área.

Embora não se possa caracterizar o processo de internacionalização da produção científica da Enfermagem brasileira apenas pela publicação em periódicos nacionais de circulação internacional, é inegável a sua importância no crescimento da Enfermagem mundial. Essa pode ser comprovada pela participação dos docentes na arbitragem de artigos e na composição de conselhos editoriais de periódicos do exterior com fator de impacto. Destacam-se ainda, a contribuição dos pesquisadores brasileiros na organização ou participação por convite em eventos científicos no exterior ou itinerante no Brasil além de comitês de sociedades científicas internacionais, a captação de financiamento em agências internacionais, os projetos conjuntos, a participação em bancas e comitês de avaliação no exterior, a orientação de pós-graduandos em outros países, a cotutela de teses, entre outros.

As ações que visam à internacionalização podem ser identificadas no aumento da mobilidade de docentes e discentes em atividades científicas, caracterizada tanto pela ida ao exterior como professor visitante, ministrante de disciplinas e cursos, realização de pós-doutoramento, doutorado sanduíche, estágios sênior e visitas técnicas, como também pelo recebimento de estrangeiros como alunos regulares dos programas, estagiários em pós-doutoramento e de intercâmbios/convênios de cooperação com reciprocidade entre docentes dos programas e das instituições de reconhecimento internacional na área, entre outras. Essas ações também se refletem nas atividades de melhoria da escrita e comunicação em inglês científico que devem ser objeto de atenção dos programas da Área de Enfermagem ${ }^{(10)}$.

No entanto, a área ainda considera necessário avançar cada vez mais na capacitação em língua inglesa para fluência e melhoria da escrita e consolidar o processo de internacionalização, com vistas a fortalecer as iniciativas de mobilidade para instituições estrangeiras com expertise em tecnologia e inovação em saúde e Enfermagem, visando à obtenção de maior impacto no avanço da sociedade do conhecimento. Também merece atenção no processo de internacionalização o incremento no desenvolvimento de pesquisas colaborativas e multicêntricas e a produção científica e tecnológica em parceria com pesquisadores estrangeiros, prática ainda restrita a poucos programas da Área de Enfermagem ${ }^{(10)}$.

Apesar dos avanços apontados na produção científica e na internacionalização da área, tem-se como desafio a transferência de conhecimento para a prática de Enfermagem e saúde, o que se constitui em pauta das discussões de área e programas, na perspectiva da prática baseada em evidência, bem como se ressalta a necessidade de avançar no desenvolvimento de tecnologia e inovação para o cuidado em saúde, gerenciamento de serviços e ensino em Enfermagem.

Desta forma, se constitui também como desafio para a área incrementar a produção científica em sintonia com as diretrizes traçadas pelos organismos/agências nacionais e internacionais e as políticas de ciência, tecnologia e inovação e a Agenda Nacional de Prioridades de Pesquisa em Saúde.

Nesse sentido é fundamental que os pesquisadores proponham "ações mais arrojadas e desenvolvimento do empreendedorismo como ferramenta para tornar mais visíveis as investigações científicas da Enfermagem, com produtos que possam ser mais eficientes para fazer avançar a prática de Enfermagem e produzir novas formas de cuidar e de gerenciar em saúde"(11). Para tanto, é necessário avanço no desenvolvimento de projetos de pesquisa para que estes promovam a inovação e possibilitem a transferência dos resultados de pesquisa para os serviços de saúde, de acordo com as políticas públicas de saúde, visando a consolidação do Sistema Único de Saúde - SUS, com promoção da qualidade de vida por meio da excelência do cuidado de Enfermagem ${ }^{(11-12)}$.

Outro aspecto em pauta nas discussões da pós-graduação brasileira e desafiante para as áreas na CAPES refere-se à interdisciplinaridade, contemplada em item específico do Documento da Área de $2013^{(10)}$ elaborado com a participação de consultores, o qual apresentamos a seguir.

O PNPD 2011-2020 destaca, em suas diretrizes, temáticas interdisciplinares, reconhecendo a importância crescente de segmentos do conhecimento e da pesquisa que exigem variadas metodologias e conceitos disciplinares para o enfrentamento de diferentes problemas. Dentre as diretrizes específicas indicadas, constam a ampliação e aprofundamento da visão interdisciplinar na formação integrada de pessoas e o estímulo às experiências interdisciplinares por parte das instituições de ensino e pesquisa ${ }^{(9)}$.

A Enfermagem é campo de conhecimento específico e profissão social que, por sua natureza, relaciona suas práticas tanto no âmbito individual quanto coletivo. Na construção de seu saber, a Enfermagem se aproximou das áreas biológico-biomédicas, sociais e humanas. Como uma disciplina prática do ramo das ciências aplicadas da área da saúde, a forte influência do modelo biomédico se fez/faz sentir tanto na construção de seu saber quanto de suas práticas ${ }^{(13)}$. Neste ínterim, o cuidado de Enfermagem, seu objeto de trabalho, em muito reflete esta influência ao se deter no corpo biológico dos indivíduos, o que reduz o espectro de atuação do ponto de vista de suas práticas.

Ao refutar essa ideia reducionista, pode-se inscrevê-la em um âmbito mais coletivo da saúde e de natureza interdisciplinar, o que representa para a Enfermagem brasileira transcender a visão fragmentada do conhecimento que dificulta a compreensão da complexidade humana ${ }^{(14)}$.

Pode-se dizer que a interdisciplinaridade, para a Enfermagem, pressupõe respeito e compromisso com a integralidade 
do homem em prol da totalidade, cuja potencialidade viabiliza interações entre diferentes campos disciplinares, permitindo situar as suas práticas a partir de modos particulares de interpretações e complexidades ${ }^{(15)}$. Assim, a Enfermagem busca uma nova postura diante do conhecimento, na medida em que, ao incorporar conceitos ampliados, estabelece importantes mudanças em busca de uma unidade do pensamento.

Para a área, a interdisciplinaridade é exercício importante, na medida em que busca conexões com outras áreas, por meio de ação dialógica na elaboração, (re)construção de novos conhecimentos com vistas à melhor apropriação de suas práticas e de seu conhecimento científico. É de conhecimento da comunidade científica, que o enfrentamento das limitações que envolvem o âmbito disciplinar exige visão mais integral, cuja resposta se apresenta basicamente a partir da equidade e eficácia.

As intervenções necessitam de conhecimentos técnico-científicos que extrapolam o campo das relações interpessoais e institucionais e, nesse sentido, é salutar que, para obter êxito, a Enfermagem se aproprie de contexto mais amplo, como meio de responder de forma resolutiva aos problemas que se apresentam às suas práticas sociais.

Situar, portanto, a interdisciplinaridade como elemento importante às práticas da Enfermagem, é identificá-la no interior de um contexto que apresenta múltiplas determinações, que são sociais, culturais, políticas, psicológicas e biológicas, que historicamente a constituem. É considerar a possibilidade de formação de profissionais com visão mais global de mundo, em contraposição à fragmentação no fazer científico.

Assim, pode-se considerar que a natureza interdisciplinar encontra, na área, potencialidades para a construção de conhecimentos no enfrentamento das diversidades que circundam o saber da profissão e suas práticas sanitárias.

Dessa forma, pode-se afirmar que a incorporação da interdisciplinaridade no campo da Enfermagem propicia a identificação e o respeito pelo núcleo de saberes que conferem a essa profissão competências e responsabilidades que podem ser partilhadas com os demais profissionais de saúde e áreas afins. No entanto, há que considerar que, ao assumir a Enfermagem num campo interdisciplinar, não se nega ou anula o aspecto disciplinar que a identifica como ciência e, muito menos, a inscreve em justaposição de saberes dentro do campo multidisciplinar. Isso impõe um desafio, de compreendê-la em seus limites e ao mesmo tempo identificá-la na sua potencialidade para a construção investigativa em direção à abertura de campo de natureza interdisciplinar.

Os desafios que se apresentam à Enfermagem enquanto prática, que também exige articulações interdisciplinares, remete às reflexões dos valores agregados ao conjunto de outras disciplinas que se aproximam decisivamente às suas práticas.

Esse conjunto disciplinar norteia e agrega potencialidades para a Enfermagem e são disciplinas que advêm de importantes áreas do conhecimento, como a das ciências naturais, ciências da vida e ciências humanas, cujas contribuições se apresentam com valor incomensurável para a qualidade de vida do ser humano, ao equilibrarem os conteúdos instrumentais que norteiam a formação científica da área, na medida em que induzem a incorporação também das questões subjetivas, como fundamentos para o desenvolvimento da atuação da Enfermagem no complexo e árido campo da saúde.

Tendo em vista as características intrinsecamente abrangentes e multidisciplinares do campo de conhecimento da Enfermagem, a organização dos programas pode se apresentar com diversas composições, guardadas, contudo, as especificidades e singularidades próprias da área.

Atualmente, dentre os 63 programas de pós-graduação stricto sensu aprovados na Área de Enfermagem, 28 (44,4\%) são multiprofissionais atendendo demanda de profissionais que atuam e têm projetos de pesquisa nas áreas de Enfermagem, da Saúde e afins.

Considera-se, assim, que a Área de Enfermagem está contribuindo para a capacitação de recursos humanos e a produção de conhecimento e práticas multi e interdisciplinares para o cuidado e gestão em saúde, permanecendo o desafio da busca da excelência e inovação no processo de transferência de conhecimento e tecnologia para o cuidado individual e coletivo à saúde humana, rumo à consolidação do $S U S^{(10)}$.

\section{MESTRADO PROFISSIONAL: UMA NOVA PÁGINA NA HISTÓRIA DA PÓS-GRADUAÇÃO EM ENFERMAGEM}

As discussões acerca da modalidade de mestrado profissional no país ampliaram-se no final da década de 80 , tomando vulto na década de 90 e concretizando-se, efetivamente nos anos 2000, inclusive no que concerne aos parâmetros de avaliação desta modalidade de formação ${ }^{(16)}$. Os primeiros cursos datam de meados dos anos de 1990, regulamentados desde 1995, por legislações que buscam diferenciar os cursos acadêmicos dos profissionais ${ }^{(17-18)}$.

Mas, de que se tratam os mestrados profissionais? Podem ser conceituados como um "tipo de formação pós-graduada que envolve uma grande diversidade de formatos específicos para o seu funcionamento. É a capacitação para a prática transformadora por meio da incorporação do método científi-

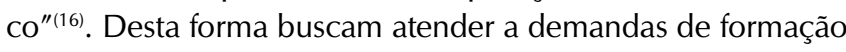
profissional de alto nível para um mercado não acadêmico, com vistas à capacitação para a "prática profissional transformadora com foco na gestão, produção ou aplicação do conhecimento, visando a solução de problemas ou proposição de inovação, por meio da incorporação do método científico e da atualização do conhecimento pertinente" ${ }^{\prime(18)}$.

Desta forma, os programas de mestrado profissional inserem-se no PNPG 2005-2010, no sentido de suprir uma lacuna na formação de profissionais altamente qualificados para o mercado público e privado, sendo reforçado este aspecto no PNPG 2011-2020(9).

O Mestrado Profissional em Enfermagem volta-se à formação de enfermeiros altamente qualificados e inseridos no mundo de trabalho, sendo alguns de caráter multiprofissional. O foco dos programas é a capacitação de profissionais para a produção de conhecimento científico-tecnológico e inovação para a geração de produtos e processos que possam transformar e qualificar a prática profissional. Espera-se nesse nível de ensino, não apenas a produção, mas também a difusão e o consumo de pesquisas e tecnologias, que possam contribuir 
para o melhor desempenho dos serviços, qualificando a assistência e/ou o ensino.

Para a Enfermagem, o mestrado profissional é uma potencialidade de qualificar o cuidado, a gestão, a educação e a própria pesquisa, que se constituem nas dimensões do fazer da profissão, pois os programas encontram-se alicerçados em princípios como aplicabilidade, flexibilidade, organicidade, inovação e valorização da experiência profissional ${ }^{(10)}$. Para tanto, a ênfase acadêmica dos mestrados profissionais deve estar alicerçada em disciplinas formativas que abordem conteúdos relacionados à atividade profissional e ao desenvolvimento do raciocínio crítico $^{(19)}$.

Nesse sentido, os mestrados profissionais em Enfermagem visam dar uma resposta às necessidades sociais, gerando produtos e processos que contribuam efetivamente para a transformação social. O primeiro Mestrado Profissional em Enfermagem teve início em 2001 na UNIFESP e tinha enfoque na área de Obstetrícia. Ofertou uma única turma que titulou quatro mestres e encerrou suas atividades em 2004, quando a última aluna defendeu sua dissertação. Em 2002 a Escola de Enfermagem Aurora de Afonso Costa, da Universidade Federal Fluminense (UFF), propôs o segundo programa de Mestrado Profissional Assistencial, sendo, por alguns anos, o único em funcionamento no país. Em 2006 foi criado o Mestrado Profissional em Enfermagem, da Universidade Estadual Paulista Júlio de Mesquita Filho - UNESP e somente em 2010 surgiu o terceiro programa no país, proposto pelo Departamento de Enfermagem da UFSC, denominado Mestrado Profissional Gestão do Cuidado de Enfermagem.

Considerando a trajetória dos mestrados profissionais em Enfermagem, observa-se um incremento nos dois últimos anos, haja vista que até 2010 existiam no país apenas os três mestrados profissionais supracitados e, atualmente, somam 14 aprovados pelo CTC/CAPES. Apesar de a maioria ser recente, esta modalidade de mestrado ocupa um espaço importante na formação de profissionais para o desenvolvimento de tecnologias, como recomendado na Política Nacional de Ciência Tecnologia em Inovação em Saúde, já que os mestrado profissional partem dos problemas identificados na realidade dos serviços, buscando soluções para estes ${ }^{(20)}$.

Dos 14 programas existentes, cinco têm nota 4 na avaliação da CAPES e nove nota 3, sendo que os critérios de avaliação têm sido discutidos pela área e aprimorados, no sentido de dar visibilidade aos mesmos e, principalmente, estabelecer indicadores de avaliação diferentes dos utilizados nos programas acadêmicos, haja vista a natureza e objetivo dos mestrados profissionais. Neste sentido, há que se considerar no processo de avaliação, entre outros aspectos, a origem dos alunos, que devem ter prioritariamente inserção no mercado de trabalho, o acompanhamento dos egressos e a parceria com os serviços dos quais os alunos são oriundos, visando avaliar o impacto destes programas na melhoria da prática profissional e dos serviços.

A preocupação com os critérios de avaliação que direcionem o mestrado profissional para as suas especificidades tem sido tema recorrente no Fórum Nacional dos Mestrados Profissionais, bem como nos dois fóruns específicos que trataram dos Mestrados Profissionais na Enfermagem. O primeiro ocorreu em Niterói/RJ, promovido pela UFF, em 2011, e o segundo em Vitória/ES, em 2012, organizado pela Universidade Federal do Espírito Santo - UFES. A mesma temática tem sido pauta dos Seminários de Acompanhamento da Pós-Graduação na Área de Enfermagem ocorridos na CAPES em 2012 e 2013, nos quais os coordenadores dos mestrados profissionais estiveram presentes.

Embora as reais especificidades do Mestrado Profissional em Enfermagem ainda sejam objeto de discussão na própria área e junto aos programas, observa-se um amadurecimento dos programas já consolidados e uma tendência mais clara dos mais recentes acerca da responsabilidade com o impacto na geração de conhecimento visando à transformação da prática de Enfermagem. Acredita-se que para alcançar este impacto, há que se pensar em novas formas de flexibilizar e inovar a pós-graduação, haja vista que os mestrados profissionais são "experiências de inovação e reinvenção das práticas acadêmicas e como tal devem ser tratados. [...] Talvez seja esse o maior desafio e fator de sedução para oferecer cursos de natureza profissionalizante: a articulação orgânica entre a prática (que o aluno tem ou almeja ter) e a teoria que alimenta e alicerça esta prática"(17). Portanto, o mestrado profissional é uma nova página na história da Pós-Graduação em Enfermagem e como tal deve ser considerado.

\section{CONSIDERAÇÕES FINAIS}

A área de Enfermagem segue crescendo em bases fortalecidas e seguras para sua sustentação como profissão alicerçada na ciência e tecnologia e com o impacto reconhecido no desenvolvimento social das diferentes regiões de nosso país, marcando contribuições efetivas para a melhoria da atenção à saúde. Sua visibilidade e inserção internacional, do mesmo modo, vêm crescendo expressivamente na conquista de espaços por experiências e projetos colaborativos da maior relevância para sua qualificação em nível avançado. Os Coordenadores da Área de Enfermagem na CAPES vêm preservando a tradição, filosofia e política da área, no desafio de conquistar avanços e atender as expectativas da comunidade compromissada com a Pós-Graduação da Enfermagem brasileira.

As políticas propostas e implementadas pelas Coordenações da Área vêm promovendo a consolidação da Enfermagem centrada no avanço da ciência, tecnologia e inovação em Enfermagem e compromisso de elevar a qualidade de vida e saúde da população e o destaque científico de nosso país.

Nesse sentido, a Área de Enfermagem na CAPES vem contribuindo para o avanço e a consolidação do conhecimento científico, tecnológico e de inovação em Enfermagem e Saúde, quer pelo desempenho demonstrado na produção científica e tecnológica avançada, quer pela formação de recursos humanos de excelência nos níveis de mestrado acadêmico, doutorado e mestrado profissional, tendo como filosofia, o respeito à diversidade e o livre debate de ideias, a elevação da qualidade de vida e saúde e a efetivação do exercício da cidadania.

A partir dos avanços e desafios da pós-graduação na Área de Enfermagem aqui apontados e amplamente discutidos com os coordenadores e cursos/programas, vislumbram-se as seguintes perspectivas futuras: 
$\checkmark$ Expansão qualitativa da pós-graduação na área com redução das assimetrias regionais;

$\checkmark$ Maior identidade e visibilidade regional e nacional do mestrado profissional na área, para qualificação dos profissionais inseridos no mercado de trabalho com desenvolvimento de tecnologia e inovação, contribuindo com a melhoria das práticas e consolidação do SUS;

$\checkmark$ Reconhecimento da excelência internacional no ensino e na pesquisa de programas de pós-graduação em Enfermagem, com alcance da nota 7 na avaliação da CAPES;

$\checkmark$ Aprimoramento dos critérios de avaliação dos cursos/ programas, em especial aqueles pertinentes ao mestrado profissional;

$\checkmark$ Ampliação do potencial da área para contribuir com a capacitação de recursos humanos e a produção de conhecimento, inovação e práticas multi e interdisciplinares para o cuidado individual e coletivo à saúde humana e a gestão dos serviços de saúde, em consonância com o PNPG 2011-2020.

\section{REFERÊNCIAS}

1. Bueno Robles LS, Díaz Heredia LP, Mejía Rojas ME, López L. Panorama de la formación doctoral en enfermería. Av Enferm 2010;28(2):134-144.

2. American Association of Colleges of Nursing. Indicators of quality in research-focused doctoral programs in nursing. J Prof Nurs [periódico na internet] 2001 Out [acesso em 06 ago 2013];18(5):289-94. Disponível em: http:// www.ncbi.nlm.nih.gov/pubmed/12434322

3. Zucco C. Relação entre pós-graduação e graduação: a pós-graduação no contexto histórico educacional. In: Discussão da Pós-Graduação Brasileira. Brasília: CAPES; 1996. p.79-96.

4. Gutiérrez MGR, Vieira TT, Almeida MCP, Elsen I, Stefanelli MC. Acompanhamento e avaliação da pós-graduação no Brasil: retrospectiva histórica da representação de enfermagem. Esc Anna Nery Rev Enferm 2001;5(2):161-72.

5. Coordenação de Aperfeiçoamento de Pessoal de Nível Superior [homepage na internet]. Avaliação da pós-graduação [acesso em 06 ago 2013]. Disponível em: http://www.capes.gov.br/images/stories/download/avaliacao/2003_020_Doc_Area.pdf

6. Coordenação de Aperfeiçoamento de Pessoal de Nível Superior [homepage na internet]. Relatório anual: avaliação continuada [acesso em 06 ago 2013]. Disponível em http://www.capes.gov.br/images/stories/download/avaliacao/DocArea_Enfermagem_Anobase2004.pdf

7. Coordenação de Aperfeiçoamento de Pessoal de Nível Superior [homepage na internet]. Documento da área 2009 [acesso em 06 ago 2013]. Disponível em: http:// www.capes.gov.br/images/stories/download/avaliacao/ ENFERMAGEM_22jun10b.pdf

8. Coordenação de Aperfeiçoamento de Pessoal de Nível Superior [homepage na internet]. Relatório de avaliação 2007-2009: trienal 2010 [acesso em 06 ago 2013]. Disponível em http://trienal.capes.gov.br/wp-content/uploads/2010/12/ENFERMAGEM-RELAT\%C3\%93RIO-DE-AVALIA\%C3\%87\%C3\%83O-FINAL-dez10.pdf

9. Coordenação de Aperfeiçoamento de Pessoal de Nível Superior. Plano Nacional de Pós-Graduação - PNPG - 20112020/CAPES. Brasília, DF: CAPES; 2010. P. 309.Vol 1.

10. Coordenação de Aperfeiçoamento de Pessoal de Nível Superior [homepage na internet]. Avaliação [acesso em 06 ago 2013]. Disponível em http://www.capes.gov.br/component/ content/article/44-avaliacao/4667-enfermagem

11. Scochi CGS, Munari DB. A pós-graduação em Enfermagem brasileira faz quarenta anos: avanços, desafios e necessidades de novos empreendimentos. Esc Anna Nery Rev Enferm 2012;16(2):215-8.

12. Erdmann AL. Rumo à consolidação da excelência na Pós-Graduação de Enfermagem no Brasil [editorial]. Acta Paul Enferm 2011;24(1):vii.

13. Oliveira MAC. A interdisciplinaridade no ensino e na pesquisa em Enfermagem [editorial]. Rev Esc Enferm USP 2012;46(2).

14. Menossi MJ, Oliveira MM, Coimbra VCC, Palha PF, Almeida MCP. Interdisciplinaridade: um instrumento para a construção de um modelo assistencial fundamentado na promoção da saúde. Rev Enferm UERJ 2005;13:252-6.

15. Matos E, Pires DEP, Campos GWS. Relações de trabalho em equipes interdisciplinares: contribuições para novas formas de organização do trabalho em saúde. Rev Bras Enferm 2010;62(6):775-81. Disponível em: http://www.scielo.br/scielo.php?pid = S0034-71672009000600010\&script $=$ sci_arttext

16. Barros EC, Valentim MC, Melo MAA. O debate sobre o mestrado profissional na Capes: trajetória e definições. RBPG 2005;2(4):124-38.

17. Fischer T. Mestrado Profissional como prática acadêmica. RBPG 2005;2(4):24-9.

18. Brasil. Ministério da Educação. Portaria normativa n. ${ }^{\circ}$ 17, de 28 de dezembro de 2009. Dispõe sobre o mestrado profissional no âmbito da Fundação Coordenação de Aperfeiçoamento de Pessoal de Nível Superior - CAPES [portaria na internet]. Diário Oficial da União $29 \mathrm{dez}$ 2009. [acesso em 05 mar 2013]. Disponível em: http:// www.capes.gov.br/images/stories/download/legislacao/ PortariaNormativa_17MP.pdf

19. Scochi CGS, Munari DB. Mestrado profissional: formação stricto senso em expansão na área de Enfermagem [editorial]. Rev Enferm UFSM [periódico na internet]. 2012 [acesso em 05 mar 2013];2(2). Disponível em: http://cascavel.ufsm.br/revistas/ojs-2.2.2/index.php/reufsm/article/ view/6254/3765.

20. Scochi CGS, Munari DB, Gelbcke FL. Mestrado Profissional em Enfermagem: possibilidades frente à Política Nacional de Ciência e Tecnologia [editorial]. Revista de Pesquisa Cuidado é Fundamental. Online [periódico na internet]. 2012 [acesso em 05 mar 2013];4(4). Disponível em: http://www.seer.unirio.br/index.php/ cuidadofundamental/article/view/2381/pdf_635 